\title{
Improving enzyme optimum temperature prediction with resampling strategies and ensemble learning
}

\author{
Japheth E. Gado',2, Gregg T. Beckham², and Christina M. Payne ${ }^{1}$ \\ ${ }^{1}$ Department of Chemical and Materials Engineering, University of Kentucky, Lexington, KY, USA \\ ${ }^{2}$ National Bioenergy Center, National Renewable Energy Laboratory, Golden, CO, USA
}

\begin{abstract}
Accurate prediction of the optimal catalytic temperature ( $\left.T_{\text {opt }}\right)$ of enzymes is vital in biotechnology, as enzymes with high $T_{\text {opt }}$ values are desired for enhanced reaction rates. Recently, a machine-learning method (TOME) for predicting $T_{\text {opt }}$ was developed. TOME was trained on a normally-distributed dataset with a median $T_{\text {opt }}$ of $37^{\circ} \mathrm{C}$ and less than five percent of $T_{\text {opt }}$ values above $85^{\circ} \mathrm{C}$, limiting the method's predictive capabilities for thermostable enzymes. Due to the distribution of the training data, the mean squared error on $\mathrm{T}_{\mathrm{opt}}$ values greater than $85^{\circ} \mathrm{C}$ is nearly an order of magnitude higher than the error on values between 30 and $50^{\circ} \mathrm{C}$. In this study, we apply ensemble learning and resampling strategies that tackle the data imbalance to significantly decrease the error on high $\mathrm{T}_{\text {opt }}$ values $\left(>85^{\circ} \mathrm{C}\right)$ by $60 \%$ and increase the overall $R^{2}$ value from 0.527 to 0.632 . The revised method, TOMER, and the resampling strategies applied in this work are freely available to other researchers as a Python package on GitHub.
\end{abstract}

\section{INTRODUCTION}

Enzymes that are stable and active at high temperatures are especially desirable for industrial applications, as they enable biochemical processes to be conducted at higher temperatures yielding faster reaction rates. Hence, researchers have long sought to develop tools for accurate in silico prediction of enzyme thermostability. Accordingly, many tools have been developed over the past two decades to predict the enzyme melting temperature $\left(T_{m}\right),^{1-3}$ the change in thermodynamic stability $(\triangle \Delta G)$ upon point mutations ${ }^{4-12}$ or the optimal growth temperature (OGT) of the source organism. ${ }^{13-21}$ Unfortunately, for prediction purposes, higher OGT or thermal stability do not necessarily indicate substantial catalytic activity at high temperatures. ${ }^{22,}{ }^{23}$ Hence, a tool that directly predicts the optimal catalytic temperature ( $\left.T_{\text {opt }}\right)$ of enzymes is desirable.

Recently, Li et al. developed a machine-learning tool, TOME (Temperature Optima for Microorganisms and Enzymes), for predicting the OGT of microorganisms and the $T_{\text {opt }}$ of enzymes. ${ }^{23}$ TOME uses a support vector regressor to predict OGT from the dipeptide composition of the proteome, and a random forest regressor to predict $\mathrm{T}_{\text {opt }}$ from the OGT and the amino acid composition. In predicting OGT, TOME achieved an $\mathrm{R}^{2}$ value of 0.88 in cross validation tests, which is superior to other published models. ${ }^{24,}{ }^{25}$ However, the $\mathrm{R}^{2}$ value of $\mathrm{T}_{\text {opt }}$ prediction was only 0.51 , providing impetus for further improvement. More recently, ${ }^{26} \mathrm{Li}$ et al. incorporated feature engineering to improve the accuracy of $\mathrm{T}_{\mathrm{opt}}$ prediction. They extracted 5,494 and 5,700 sequence features, using the packages, iFeature and UniRep, respectively. ${ }^{27,} 28$ However, these features did not provide a significant improvement in performance compared to using only the amino acid composition and OGT, even when deep learning was applied. As a result, the authors concluded that more informative features, such as features from the threedimensional structure, may be necessary to markedly improve $T_{\text {opt }}$ prediction performance. Yet, a tool 
that accurately predicts $T_{\text {opt }}$ from sequence-data alone remains valuable to the biotechnology community, since it can be readily applied to the vast number of proteins in the databases that lack structural characterizations.

In this work, we sought to improve the accuracy of $\mathrm{T}_{\text {opt }}$ prediction, not by customary feature engineering, but by mitigating the adverse impact of the non-uniform distribution of the training data used in the machine learning model. It is recognized that an imbalanced data distribution is highly unfavorable in machine learning problems, as it biases the learning algorithms towards the abundant data regions at the expense of the poorly sampled regions, and, thus, leads to higher error on the rare values and overall sub-optimal model performance. ${ }^{29-31}$ In classification problems, data imbalance has been extensively studied, and numerous techniques for dealing with imbalance problems have been proposed. ${ }^{32,33}$ These methods are generally classified into three groups: algorithm-level methods, which specifically modify the learning algorithm to address the bias; data-level methods, which resample the data in a preprocessing step to decrease the unevenness of the data; and hybrid methods, which combine both algorithm- and data-level methods. ${ }^{30,34}$ Data-level methods modify the data distribution primarily by either undersampling the majority class, oversampling the minority class, or a combination of both. ${ }^{34}$ Researchers have developed multiple resampling methods for classification problems such as neighborhood cleaning rule (NCL), ${ }^{35}$ synthetic minority oversampling technique (SMOTE), ${ }^{36}$ selective preprocessing of imbalanced data (SPIDER), ${ }^{37}$ and majority undersampling technique (MUTE). ${ }^{38}$ The combination of resampling strategies with ensemble learning (the integration of the outcomes of multiple base models) has proven remarkably successful in dealing with class imbalance. ${ }^{34,39,40}$

On the contrary, less attention has been paid to imbalance in regression problems. ${ }^{30,32} \mathrm{Few}$ methods have been proposed for working with imbalanced distributions in regression domains including: SMOTE for regression (SMOTER), ${ }^{41}$ SMOGN, ${ }^{42}$ meta learning for utility maximization (MetaUtil), ${ }^{43}$ resampled bagging (REBAGG), ${ }^{44}$ and weighted relevance-based combination strategy (WERCS). ${ }^{45}$ In many bioinformatic and cheminformatic supervised-learning regression problems, the data often follows a normal distribution, and the rare extreme values may be more important to the user than the abundant values centered about the median of the distribution. For example, in predicting $T_{\text {opt }}$ for practical applications, higher $T_{\text {opt }}$ values are generally more relevant since thermostable enzymes are desired for enhanced biochemical reaction rates. Still, a majority of studies do not address the issue of data imbalance, ${ }^{10}, 11,46,47$ resulting in models with reduced predictive accuracy at tails of the normal distribution. ${ }^{32,45}$ Additionally, standard metrics used in assessing regression model performance, such as mean squared error (MSE) and mean absolute deviation (MAD), are heavily biased towards the abundant values centered about the median so that the reported performance fails to capture the poorer performance on rare values at the tails of the distribution. ${ }^{48}$ Consequently, a model could demonstrate excellent performance on non-uniform datasets and, yet, might have little ability to accurately predict extreme values.

In this study, we apply resampling and ensemble methods to enzyme $T_{\text {opt }}$ prediction. Our results show that without resampling (i.e., TOME), the error (MSE) in predicting high temperature values $\left(>65^{\circ} \mathrm{C}\right.$ ) was more $500 \%$ higher than the error in predicting $\mathrm{T}_{\text {opt }}$ values centered about the median $\left(30-50^{\circ} \mathrm{C}\right)$. By applying resampling strategies alone, without the introduction of new features, we were able to reduce the error on high temperature values $\left(>65^{\circ} \mathrm{C}\right)$ by more than $50 \%$ and, consequently, increase the overall performance $\left(R^{2}\right)$ by $20 \%$. We make available the machine-learning tool for improved $T_{\text {opt }}$ prediction, TOMER (Temperature Optima for Enzymes with Resampling), through GitHub. We anticipate TOMER will prove valuable in accurately predicting $T_{\text {opt }}$ values of industrially-relevant, thermostable enzymes. To facilitate minimizing the impact of data imbalance in other regression applications, we have also provided the resampling strategies employed here as a Python package, resreg (Resampling for Regression). 


\section{METHODS}

\subsection{Dataset and machine learning implementation}

The dataset used in training TOMER was obtained from Li et al., consisting of 2,917 enzymes with experimental $\mathrm{T}_{\mathrm{opt}}$ measurements and OGT data from the BRENDA database. ${ }^{26,49}$ Throughout this work, all machine learning regressors were trained on the same 21 features used in TOME, which include the frequencies of the 20 amino acids and the OGT. The features were normalized by subtracting the mean and dividing by the standard deviation before fitting the regressors. Machine learning was implemented with the scikit-learn package $(v 0.21 .2)^{50}$ in Python (v3.6.6).

\subsection{Evaluation of performance}

In evaluating the performance of the regressors, we did not use the conventional $k$-fold cross validation technique. Since the data are normally distributed, randomly splitting the data into folds will result in similarly imbalanced folds and, as a result, the performance metrics ( $\left.R^{2}, M S E\right)$ will overly weight the frequent data and will not sufficiently capture the performance at the distribution tails. Hence, we evaluated performance of the regressors on a testing set that was nearly uniformly distributed. A uniform testing set was formed by splitting the entire dataset into five bins based on the target values ( $\left.T_{\text {opt }}\right)$. Then, 70 samples were randomly selected from each bin to constitute the testing set, with the remaining data forming the training set (Table 1, Figure 1A). We selected only 70 samples from each bin so that at least half of the data in the smallest bin $\left(85-120^{\circ} \mathrm{C}\right)$ was used in training. This way, $88 \%$ of the entire dataset was used in training (2,567 samples) and $12 \%$ in testing (350 samples). The dataset was repeatedly split into training and testing sets 50 times, and each time, resampling strategies were applied to the training set before fitting the regressors. The performance on the testing set was measured as an average over the 50 iterations, i.e., Monte Carlo cross validation (MCCV). ${ }^{51}$

\section{Table 1. Formation of a uniform testing set by selecting equal samples from five bins.}

\begin{tabular}{|c|c|c|c|c|c|}
\hline Bins & Range $\left({ }^{\circ} \mathrm{C}\right)$ & $\begin{array}{c}\text { Samples } \\
\text { in bin }\end{array}$ & $\begin{array}{c}\text { Percent of } \\
\text { total dataset }\end{array}$ & $\begin{array}{c}\text { Testing } \\
\text { size }\end{array}$ & $\begin{array}{c}\text { Training } \\
\text { size }\end{array}$ \\
\hline $0-30$ & $0 \leq \mathrm{y}<30$ & 461 & $15.8 \%$ & 70 & 391 \\
\hline $30-50$ & $30 \leq \mathrm{y}<50$ & 1427 & $48.9 \%$ & 70 & 1357 \\
\hline $50-65$ & $50 \leq \mathrm{y}<65$ & 519 & $17.8 \%$ & 70 & 449 \\
\hline $65-85$ & $65 \leq \mathrm{y}<85$ & 361 & $12.4 \%$ & 70 & 291 \\
\hline $85-120$ & $85 \leq \mathrm{y} \leq 120$ & 149 & $5.1 \%$ & 70 & 79 \\
\hline & Total & 2,917 & $100 \%$ & $\begin{array}{c}350 \\
(12 \%)\end{array}$ & $\begin{array}{c}2,567 \\
(88 \%)\end{array}$ \\
\hline
\end{tabular}

Four metrics were used to assess the predictive performance. The coefficient of determination $\left(R^{2}\right)$ on a uniformly-distributed test set was used to assess the overall performance, and was the primary metric for selecting the best resampling strategy. Both real and predicted $T_{\text {opt }}$ values were converted to categorical values (0-30 is $1,30-50$ is $2,50-65$ is 3, etc., see Table 1), and the Matthew's correlation coefficient $(\mathrm{MCC})^{52}$ was determined as for a multiclass classification problem. ${ }^{53}$ The mean squared error (MSE) was calculated for each bin to evaluate the variation in the performance across the range of $T_{\text {opt }}$ values and to examine the error on rare high values relative to the error on abundant values. Finally, we measured the $F_{1}$ score as a way to assess the predictive performance on high $T_{\text {opt }}$ values at the distribution's tail $\left(\geq 65^{\circ} \mathrm{C}\right)$. The $F_{1}$ score, which is the weighted harmonic mean of precision and recall, is typically a classification performance metric, but has been adapted for regression problems. ${ }^{41,48}$ For regression, recall and precision has been defined as: ${ }^{48}$ 

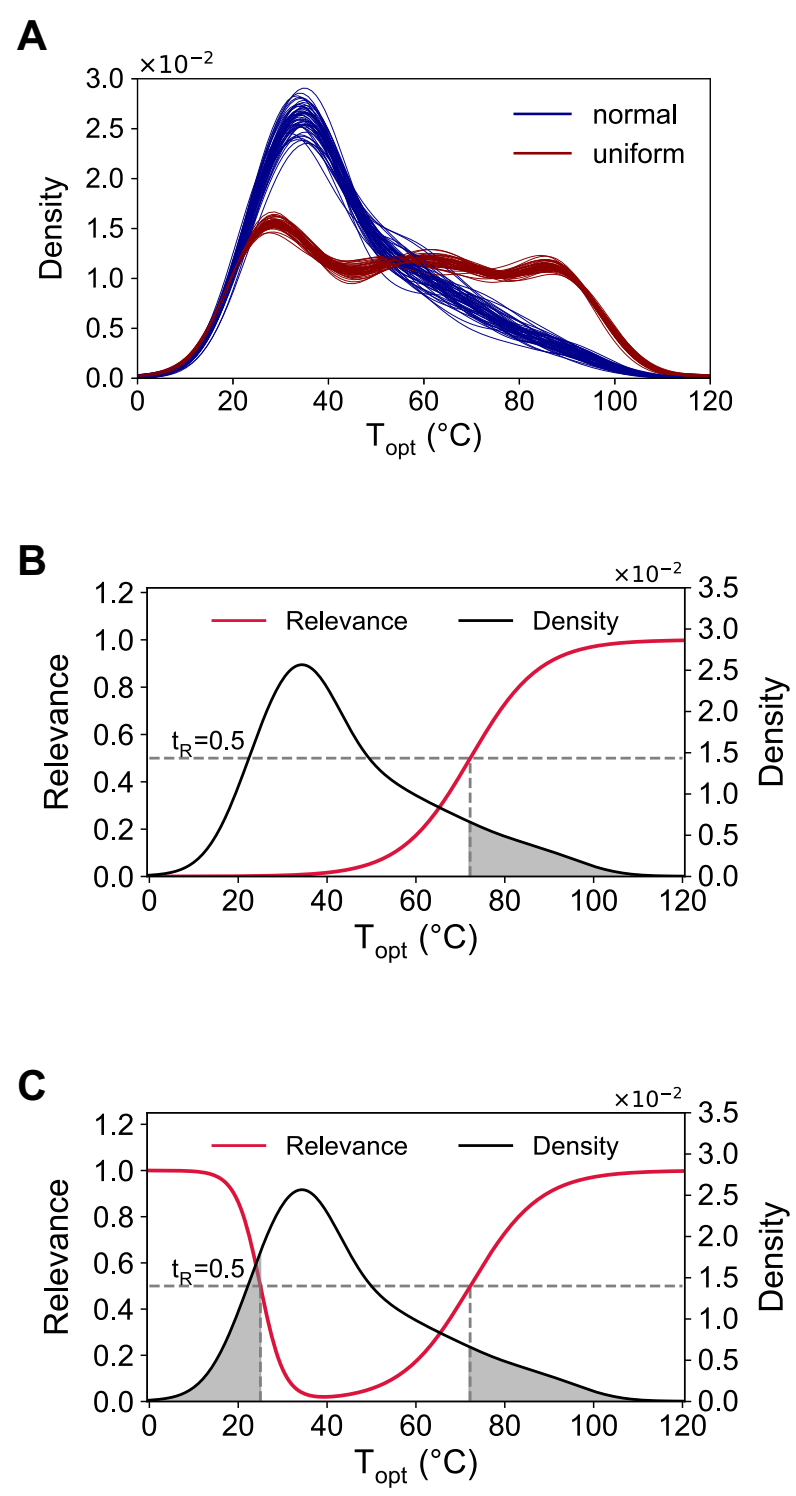

Figure 1. Distribution of $T_{\text {opt }}$ values in the dataset of 2,917 proteins. The density plots were derived using a Gaussian kernel density estimation (KDE). (A) Distribution of testing set in 50 iterations of Monte Carlo cross validation. A normally-distributed testing set formed by random selection of 350 samples is shown in blue, and the nearly uniform testing set formed by selecting 70 samples from five bins is shown in red. (B) A one-sided sigmoid relevance function that maps $T_{\text {opt }}$ values to relevance values between 0 and 1 (left-hand $y$-axis). By setting the value of $c$ in the relevance function (eq 5) to the $90^{\text {th }}$ percentile (72.2), Topt values greater than $72.2^{\circ} \mathrm{C}$ form the rare domain (shaded region) and all other values form the normal domain. The $T_{\text {opt }}$ distribution density is shown on the right-hand -axis. (C) A two-sided relevance function mapping $T_{\text {opt }}$ values to relevance values between 0 and 1 . By setting the values of $C_{L}$ and $C_{H}$ in the relevance function (eq 6) to be the $10^{\text {th }}$ and $90^{\text {th }}$ percentile ( 25 and 72.2 , respectively), $T_{\text {opt }}$ values less than $25^{\circ} \mathrm{C}$ and greater than $72.2^{\circ} \mathrm{C}$ form the rare domain, and the complement of the rare domain forms the normal domain. The $T_{o p t}$ distribution density is shown on the right-hand $y$-axis. 


$$
\begin{gathered}
\text { precision }=\frac{\sum_{\phi\left(\hat{y}_{i}\right) \geq t_{R}}\left(\alpha\left(y_{i}, \hat{y}_{i}\right) \times \phi\left(\hat{y}_{i}\right)\right)}{\sum_{\phi\left(\hat{y}_{i}\right) \geq t_{R}} \phi\left(\hat{y}_{i}\right)} \\
\text { recall }=\frac{\sum_{\phi\left(y_{i}\right) \geq t_{R}}\left(\alpha\left(y_{i}, \hat{y}_{i}\right) \times \phi\left(y_{i}\right)\right)}{\sum_{\phi\left(y_{i}\right) \geq t_{R}} \phi\left(y_{i}\right)}
\end{gathered}
$$

where $y_{i}$ and $\hat{y}_{i}$ are the true and predicted $\mathrm{T}_{\mathrm{opt}}$ values, respectively; $\phi\left(\hat{y}_{i}\right)$ is the relevance function which maps the target values to a relevance scale from 0 to 1 (discussed below); $t_{R}$ is the relevance threshold that forms the subdomain of relevant rare values, and $\alpha\left(y_{i}, \hat{y}_{i}\right)$ is a function that defines the accuracy of a prediction. Hence, the precision and recall are measures of the predictive accuracy on rare values, weighted by the relevance function. The accuracy function was defined as: ${ }^{48}$

$$
\alpha\left(y_{i}, \hat{y}_{i}\right)=I\left(L\left(y_{i}, \hat{y}_{i}\right) \leq t_{L}\right) \times\left(1-\exp \left(\frac{-k\left(L\left(y_{i}, \hat{y}_{i}\right)-t_{L}\right)^{2}}{t_{L}{ }^{2}}\right)\right)
$$

where $L\left(y_{i}, \hat{y}_{i}\right)$ is the loss function and is equal to the absolute error of the prediction; $I$ is the indicator function, which returns 1 if the absolute error is less than a threshold loss, $t_{L}$, but zero otherwise; and $k$ is an integer that defines the steepness of the accuracy curve. We set $k$ to be $10^{4}$ and $t_{L}$ to be 5 so that predictions within error limits of $5^{\circ} \mathrm{C}$ are regarded as accurate. A right-sided relevance function was used, with $t_{R} \geq 0.5$ for all $y \geq 65$ (see eq 5 and eq6), and the $F_{1}$ score was calculated from precision and recall as:

$$
F_{1}=\frac{2 \times \text { precision } \times \text { recall }}{\text { precision }+ \text { recall }}
$$

\subsection{The relevance function}

In classification problems, resampling strategies can be readily applied since the target values are clearly divided into discrete classes. Resampling is not as straightforward in regression problems, however, since the target variable is continuous. The concept of a relevance function was introduced in previous works to simplify resampling in regression problems. ${ }^{41,48,54}$ The relevance function is a userdefined function that maps the domain of target values to a scale from 0 to 1 , where 1 indicates maximum relevance. By specifying a relevance function, $\phi(y)$, and a relevance threshold, $t_{R}$, the domain of target values, $D$, can be split into two sub-domains: a domain of rare values, $D_{R}$, which is of greater importance to the user, and the domain of normal values, $D_{N}$ (Figure 1B and 1C). Consequently, $D_{R}$ and $D_{N}$ can be resampled accordingly.

In this work, we use a sigmoid relevance function defined as: ${ }^{48}$

$$
\phi(y)=\frac{1}{1+\exp (-s(y-c))}
$$

where $y$ is the target variable, and $s$ and $c$ are constants that determine the shape and center of the sigmoid, respectively. By defining $s$ as $\pm \frac{\log \left(10^{4}-1\right)}{|c|}$, it follows that $s>0$ implies that $\phi(y) \geq 0.5$ for all $y \geq$ $c$, and $s<0$ implies that $\phi(y) \geq 0.5$ for all $y \leq c .{ }^{48}$ Hence, $c$ can be specified so that extreme target values beyond $c$ have relevance values above a threshold $\left(t_{R}\right)$ of 0.5 and, thus, form the domain of rare values, $D_{R}$. Otherwise stated, $D_{R}=\left\{y: \phi(y) \geq t_{R}\right\}$ and $D_{N}=\left\{y: \phi(y)<t_{R}\right\}$. Equation 5 is used to determine $\phi(y)$ in the case that the rare domain is formed from extreme values at the left or right of the normal distribution (one-sided). For a two-sided rare domain formed from both left and right extremes, we define the relevance function as:

$$
\phi(y)=\frac{1}{1+\exp \left(-\left|s_{L}\right|\left(y-c_{L}\right)\right)}+\frac{1}{1+\exp \left(\left|s_{H}\right|\left(y-c_{H}\right)\right)}
$$

where the subscripts, $\mathrm{L}$ and $\mathrm{H}$, indicate low and high extreme values, respectively (Figure $1 \mathrm{~B}$ and $\mathrm{C}$ ). 


\subsection{Resampling strategies}

Having defined a relevance function to split the dataset into a rare and normal domain, we tested several resampling methods that alter the lopsidedness of the rare domain, relative to the normal domain. The resampling methods were applied to the training set to mitigate the adverse effects of the data imbalance, and then a random forest regressor with default settings was fitted to the resampled training set. We adapted and implemented the following resampling strategies in this work: random oversampling (RO), introduction of Gaussian noise (GN), synthetic minority oversampling technique for regression (SMOTER), weighted relevance-based combination strategy (WERCS), and WERCS with Gaussian noise (WERCS-GN). ${ }^{41,45}$ We give a brief description of these methods below. See the Supporting Information for the pseudocode of these methods.

\subsubsection{Random oversampling (RO)}

With the random oversampling strategy, ${ }^{45,55}$ the rare values are oversampled by duplicating randomly selected data points, while the normal values are left unchanged. The amount of oversampling is to be specified by the user and can significantly affect the results. Branco et al. suggested two automatic methods of oversampling: balance and extreme. ${ }^{45}$ The balance option oversamples the rare domain so that it is equal in size to the normal domain. The extreme option oversamples the rare domain so that the proportion of the size of the rare domain to the size of the normal domain is reversed. For example, if the normal domain is five times larger than the rare domain, the extreme option oversamples the rare domain so that it is five times larger than the normal domain. Here, we introduced a new automatic oversampling method that is intermediate between balance and extreme, which we dub "average". According to the method selected, the size of the rare domain after oversampling, $\left|D_{R}^{\text {new }}\right|$, is determined from the size of the rare and normal domain before resampling $\left(\left|D_{R}\right|\right.$ and $\left|D_{N}\right|$, respectively) as follows:

$$
\begin{array}{ll}
\text { balance: } & \left|D_{R}^{\text {new }}\right|=\left|D_{N}\right| \\
\text { extreme: } & \left|D_{R}^{\text {new }}\right|=\frac{\left|D_{N}\right|^{2}}{\left|D_{R}\right|} \\
\text { average: } & \left|D_{R}^{\text {new }}\right|=\frac{1}{2}\left(\left|D_{N}\right|+\frac{\left|D_{N}\right|^{2}}{\left|D_{R}\right|}\right)
\end{array}
$$

Additionally, the values of $c_{L}$ and $c_{H}$, which determine the points at which the target value is split to normal and rare values, can have significant effects on the performance. Hence, we implemented a grid search to determine the optimal combination of hyperparameters for the resampling strategies. We defined the hyperparameter space as $c_{L} \in(25,30$, None $), c_{H} \in(72.2,60)$, and method $\in$ (balance, average, extreme) (Table 2). The values for $c_{L}$ correspond to the $10^{\text {th }}$ and $20^{\text {th }}$ percentile of $T_{\text {opt }}$, and the values of $c_{H}$ correspond to the $90^{\text {th }}$ and $80^{\text {th }}$ percentile, respectively. A right-sided rare domain is indicated by $c_{L}=$ None (Figure 1B and $1 \mathrm{C}$ ).

\subsubsection{Synthetic minority oversampling technique for regression (SMOTER)}

Applying the SMOTER strategy undersamples the normal values and oversamples the rare values by generating synthetic data points through interpolation between each rare value and a random selection of one of its k-nearest neighbors. ${ }^{41,42,55}$ The feature vector and target value of a synthetic instance, $X_{2}$ and $y_{2}$, respectively, are determined as follows: ${ }^{41}$

$$
\begin{gathered}
X_{2}=X_{1}+r\left(X_{n n}-X_{1}\right) \\
y_{2}=\frac{y_{1} \cdot d_{n n}+y_{n n} \cdot d_{1}}{d_{n n}+d_{1}}
\end{gathered}
$$


Table 2. Hyperparameters of resampling strategies tested with a grid search

\begin{tabular}{|c|c|}
\hline Stra & Hyperparameter range \\
\hline Random oversampling $(\mathrm{RO})^{45,55}$ & $\begin{array}{l}\mathrm{CL}=(25,30, \text { None }), \mathrm{CH}=(72.2,60), \text { method }=\text { (balance, average, } \\
\text { extreme) }\end{array}$ \\
\hline $\begin{array}{l}\text { Synthetic minority oversampling } \\
\text { technique for regression (SMOTER) }{ }^{41,42,55}\end{array}$ & $\begin{array}{l}\mathrm{C} L=(25,30, \text { None }), \mathrm{CH}_{H}=(72.2,60), \text { method = (balance, average, } \\
\text { extreme }), \mathrm{k}=(5,10,15)\end{array}$ \\
\hline Introduction of Gaussian noise $(\mathrm{GN})^{42,45,55}$ & $\begin{array}{l}\mathrm{CL}=(25,30, \text { None }), \mathrm{CH}=(72.2,60), \text { method }=\text { (balance, average, } \\
\text { extreme }), \delta=(0.1,0.5,1.0)\end{array}$ \\
\hline $\begin{array}{l}\text { Weighted relevance-based combination } \\
\text { strategy (WERCS })^{45}\end{array}$ & $\begin{array}{l}C_{L}=(25,30, \text { None }), C_{H}=(72.2,60), \text { over }=(0.5,0.75), \text { under }=(0.5 \text {, } \\
0.75)\end{array}$ \\
\hline $\begin{array}{l}\text { Weighted relevance-based combination } \\
\text { strategy with introduction of Gaussian } \\
\text { noise (WERCS-GN) }{ }^{42,45,55}\end{array}$ & $\begin{array}{l}\mathrm{CL}=(25,30, \text { None }), \mathrm{CH}=(72.2,60), \text { over }=(0.5,0.75), \text { under }=(0.5, \\
0.75), \delta=(0.1,0.5,1.0)\end{array}$ \\
\hline $\begin{array}{l}\text { Resampled } \\
\text { oversamplin }\end{array}$ & $\begin{array}{l}\left.\mathrm{C}_{\mathrm{L}}=(25,30, \text { None }), \mathrm{CH}_{\mathrm{H}}=(72.2,60), \text { method }=\text { (balance, variation }\right), \\
\mathrm{s}=(300,600)\end{array}$ \\
\hline $\begin{array}{l}\text { Resampled bagging } \\
\text { (BAGG-SMT) }^{41,44,55}\end{array}$ & $\begin{array}{l}\mathrm{CL}=(25,30, \text { None }), \mathrm{CH}=(72.2,60), \text { method }=(\text { balance, variation }), \\
\mathrm{k}=(5,10,15), \mathrm{s}=(300,600)\end{array}$ \\
\hline $\begin{array}{l}\text { Resampled bagging with introduction of } \\
\text { Gaussian noise (BAGG-GN) }{ }^{44,55}\end{array}$ & $\begin{array}{l}\mathrm{CL}=(25,30, \text { None }), \mathrm{CH}_{H}=(72.2,60), \text { method }=\text { (balance, variation), } \\
\delta=(0.1,0.5,1.0), \mathrm{s}=(300,600)\end{array}$ \\
\hline $\begin{array}{l}\text { Resampled bagging with WERCS (BAGG- } \\
\text { WR) }\end{array}$ & $\begin{array}{l}\mathrm{CL}=(25,30, \text { None }), \mathrm{CH}=(72.2,60), \text { over }=(0.5,0.75), \text { under }=(0.5, \\
0.75), \mathrm{s}=(300,600)\end{array}$ \\
\hline $\begin{array}{l}\text { Resampled bagging with WERCS-GN } \\
(\text { BAGG-WRGN) })^{44,45,55}\end{array}$ & $\begin{array}{l}\mathrm{C} L=(25,30, \text { None }), \mathrm{CH}_{H}=(72.2,60), \text { over }=(0.5,0.75), \text { under }=(0.5, \\
0.75), \delta=(0.1,0.5,1.0), \mathrm{s}=(300,600)\end{array}$ \\
\hline
\end{tabular}

where $X_{1}$ is the feature vector of an instance in $D_{R}, X_{n n}$ is one of $k$-nearest neighbors of $X_{1}, r \in[0,1]$ is a random number, $y_{1}$ and $y_{n n}$ are the target values of $X_{1}$ and $X_{n n}$, respectively, and $d_{1}$ and $d_{n n}$ are the Euclidean distances between $X_{2}$ and $X_{1}$, and between $X_{2}$ and $X_{n n}$, respectively. The amount of undersampling and oversampling was automatically determined according to the following options:

$$
\begin{array}{ll}
\text { balance: } & \left|D_{N}^{\text {new }}\right|=\left|D_{R}^{\text {new }}\right|=\frac{\left|D_{N}\right|+\left|D_{R}\right|}{2} \\
\text { extreme: } & \left|D_{N}^{\text {new }}\right|=\left|D_{R}\right| \\
& \left|D_{R}^{\text {new }}\right|=\left|D_{N}\right| \\
\text { average: } & \left|D_{N}^{\text {new }}\right|=\frac{1}{2}\left(\frac{\left|D_{N}\right|+\left|D_{R}\right|}{2}+\left|D_{R}\right|\right) \\
& \left|D_{R}^{\text {new }}\right|=\frac{1}{2}\left(\frac{\left|D_{N}\right|+\left|D_{R}\right|}{2}+\left|D_{N}\right|\right)
\end{array}
$$

Optimal hyperparameters were similarly determined by a grid search (Table 2).

\subsubsection{Introduction of Gaussian noise (GN)}

The GN strategy is identical to SMOTER in every way except that synthetic points are generated by addition of Gaussian noise rather than interpolation. ${ }^{42,45,55}$ Noise based in $N(0, \delta \times \operatorname{std}(a))$ is separately added to each feature and to the target value of a rare instance, where $\operatorname{std}(a)$ is the standard deviation of the attribute (i.e., feature or target value), and $\delta$ is a user-defined parameter that determines the amplitude of the noise.

\subsubsection{Weighted relevance-based combination strategy (WERCS)}

Rather than using a relevance threshold to split the data into rare and normal domains as with the previous strategies, the WERCS strategy uses the relevance values as weights to select data points for undersampling and oversampling. ${ }^{45}$ The data are oversampled and then undersampled by selecting instances to be duplicated and instances to be removed, respectively. Selection for oversampling and 
undersampling is performed using probabilities determined from the relevance function. For each target value in the dataset, $y_{i}$, we defined the probability that the value is selected for oversampling or undersampling ( $p_{i}^{\text {over }}$ and $p_{i}^{\text {under }}$, respectively) by eq 9 and eq 10 .

$$
\begin{gathered}
p_{i}^{\text {over }}=\frac{\phi\left(y_{i}\right)}{\sum_{i=1}^{N} \phi\left(y_{i}\right)} \\
p_{i}^{\text {under }}=\frac{1-\phi\left(y_{i}\right)}{\sum_{i=1}^{N}\left(1-\phi\left(y_{i}\right)\right)}
\end{gathered}
$$

Hence, rare values with higher relevance are more likely to be selected for oversampling and less likely to be selected for undersampling. The amount of oversampling and undersampling are hyperparameters to be specified by the user in percent (over and under, respectively).

\subsubsection{WERCS with Gaussian noise (WERCS-GN)}

We modified the WERCS strategy by adding Gaussian noise to the values selected for oversampling by the WERCS strategy. Hence, with WERCS-GN, oversampling is done with synthetic data, instead of by duplicating data points.

\subsection{Combination of resampling strategies with ensemble learning}

Ensemble learning involves training different learners and combining their output to generate a final prediction that is more accurate than the individual learners. Branco et al. developed the resampled bagging algorithm (REBAGG) for implementing resampling and bagging in imbalanced regression problems. ${ }^{44}, 56$ In this work, we applied an adaptation of the REBAGG algorithm to the prediction of $T_{\text {opt }}$ values, by implementing the resampling methods described previously in the REBAGG algorithm (See the Supporting Information for the pseudocode).

First, the dataset is split into rare and normal domains, $D_{R}$ and $D_{N}$, using the relevance function, as described previously. Then $m$ models are trained on separately resampled bootstrap samples of $s$ items from the training dataset. Two modes of the REBAGG method are applied: balance or variation mode. In balance mode, an equal number of samples, $s / 2$, is randomly drawn from $D_{R}$ and $D_{N}$. In the variation mode, however, $p \times s$ samples are drawn from $D_{R}$, and $(1-p) \times s$ samples are drawn from $D_{N}$, where $p$ is a randomly selected number from the set, $(1 / 3,2 / 5,1 / 2,3 / 5,2 / 3)$. Hence, in the variation mode, the $m$ models are trained on data that may contain either fewer, equal, or more rare samples than normal samples. If the number of samples to be drawn from $D_{R}$ is greater than $\left|D_{R}\right|$, then the extra samples are derived by oversampling the rare domain using RO, SMOTER, or GN, resampling methods as described previously. We refer to the REBAGG method in combination with these resampling methods as BAGGRO, BAGG-SMT, and BAGG-GN, respectively. A similar combination of REBAGG with WERCS and WERCS-GN (referred to as BAGG-WERCS and BAGG-WRGN) were also implemented. With BAGGWERCS and BAGG-WRGN, the data are resampled without splitting into rare and normal domains, as in the WERCS and WERCS-GN methods. Then, s samples are drawn from the resampled data for training a model in the ensemble. With these resampled bagging strategies, the resampling step is independently repeated for all $m$ models with replacement. Finally, each model is applied to the testing set, and the final prediction is determined by averaging the predictions of all $m$ models. We used a decision tree regressor with default settings as the base regressor and set $m$ to be 100 . Other hyperparameters were optimized based on the values shown in Table 2. 


\section{RESULTS AND DISCUSSION}

\subsection{Resampling strategies significantly improve predictive performance}

In this work, we applied machine learning to predict the $T_{\text {opt }}$ of 2,917 enzymes. ${ }^{23}$ The target values follow a normal distribution that creates a problem of data imbalance. Although the $T_{\text {opt }}$ values range from 0 to $120^{\circ} \mathrm{C}$, about half of the values fall within 30 to $50^{\circ} \mathrm{C}$, and high temperature data are scarce (Table 1). To deal with this data imbalance, we implemented ten strategies that abate the imbalance by resampling the training data. For each strategy, we tested several hyperparameters with a grid search (Table 2) and selected the hyperparameter combination that yielded the highest average $\mathrm{R}^{2}$ value on a uniformly-distributed testing set (Table 3). Without resampling the training data (i.e., TOME), the average $\mathrm{R}^{2}$ value over $50 \mathrm{MCCV}$ iterations was 0.527 . However, the best performance of the resampling strategies ranged from $0.567(\mathrm{RO})$ to 0.632 (BAGG-RO). Similarly, all resampling strategies yielded significantly higher $F_{1}$ scores $(>0.178)$ and MCC values $(>0.235)$ compared to TOME, which had an F1 score of 0.137 and an MCC score of 0.212 (Figure 2). These results demonstrate that the resampling strategies improve the predictive performance on high $\mathrm{T}_{\text {opt }}$ values $\left(>65^{\circ} \mathrm{C}\right)$, as illustrated by the higher $\mathrm{F} 1$ scores, and lead to superior overall performance, as illustrated by the higher $\mathrm{R}^{2}$ and $\mathrm{MCC}$ values. It is important to note that some hyperparameter combinations of the resampling strategies led to a reduction in the predictive performance compared to the model that was trained on non-resampled data (TOME) (Figure S1). Hence, it is imperative that one test a sufficiently wide range of hyperparameters to determine the optimal hyperparameter combination.

Table 3: Best hyperparameter combination for each resampling strategy yielding the highest $\mathrm{R}^{2}$ values as determined by a grid search.

\begin{tabular}{|c|c|}
\hline Strategy & Hyperparameter \\
\hline $\mathrm{RO}$ & $\mathrm{CL}_{\mathrm{L}}=$ None, $\mathrm{CH}_{\mathrm{H}}=60.0$, method=balance \\
\hline SMOTER & $\mathrm{CL}_{\mathrm{L}}=$ None, $\mathrm{C}_{\mathrm{H}}=60.0$, method=average, $\mathrm{k}=10$ \\
\hline GN & $\mathrm{CL}_{\mathrm{L}}=$ None, $\mathrm{CH}_{\mathrm{H}}=72.2$, method=balance,$\delta=0.5$ \\
\hline WERCS & $\mathrm{CL}_{\mathrm{L}}=$ None, $\mathrm{CH}_{\mathrm{H}}=72.2$, over $=0.5$, under $=0.5$ \\
\hline WERCS-GN & $\mathrm{C}_{\mathrm{L}}=$ None, $\mathrm{C}_{\mathrm{H}}=72.2$, over $=0.5$, under $=0.5, \delta=0.1$ \\
\hline BAGG-RO & $\mathrm{CL}=$ None, $\mathrm{CH}_{\mathrm{H}}=72.2$, method=variation, $\mathrm{s}=600$ \\
\hline BAGG-SMT & $\mathrm{CL}=$ None, $\mathrm{CH}_{H}=72.2$, method=variation, $\mathrm{k}=5, \mathrm{~s}=600$ \\
\hline BAGG-GN & $\mathrm{CL}=$ None, $\mathrm{CH}_{\mathrm{H}}=72.2$, method=variation $, \delta=1.0, \mathrm{~s}=600$ \\
\hline BAGG-WERCS & $\mathrm{CL}=25.0, \mathrm{CH}_{\mathrm{H}}=72.2$, over $=0.5$, under $=0.75, \mathrm{~s}=600$ \\
\hline BAGG-WRGN & $\mathrm{CL}=25.0, \mathrm{CH}_{\mathrm{H}}=72.2$, over $=0.75$, under $=0.75, \delta=0.1, \mathrm{~s}=600$ \\
\hline
\end{tabular}

From the results shown in Figure 2A-C, we observed that resampling by simple duplication of rare values, as is done in the random oversampling strategy (RO), led to lower $R^{2}, F_{1}$, and $M C C$ values than the strategies that oversample rare values by using the relevance as weights (WERCS, WERCS-GN), or by generating synthetic data through interpolation (SMOTER) or addition of noise (GN, WERCS-GN). However, this trend was not observed when the resampling methods were combined with bagging (BAGG-RO, BAGG-SMT, BAGG-GN, BAGG-WERCS, BAGG-WRGN). We anticipate that duplication performs worse than generating synthetic values because duplication causes the learning algorithms to overfit to the replicated values. Introducing synthetic values, on the other hand, would cause the algorithms to be more general in the rare data region. ${ }^{32,36,57}$ Our results indicate that generating synthetic values does not outperform duplication techniques when combined with bagging in the REBAGG strategy, likely because aggregating multiple learners overcomes the overfitting that arises due to replicated values. 
A

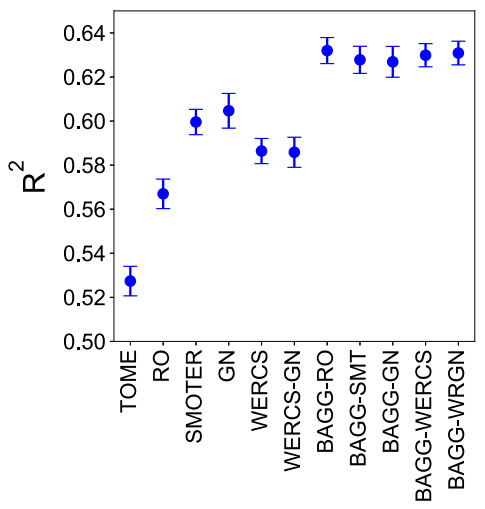

B

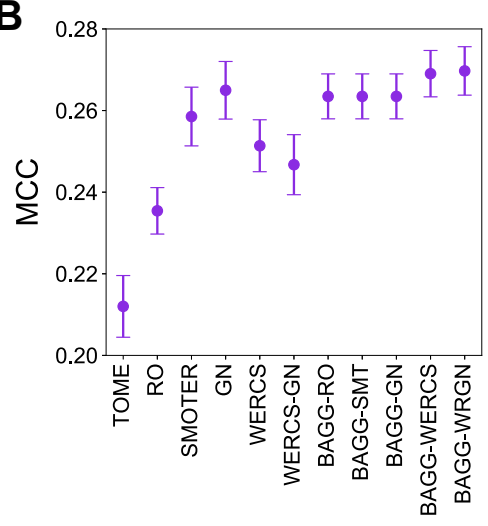

C

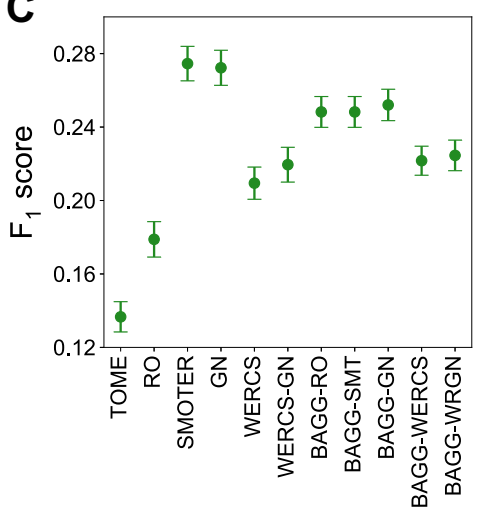

D

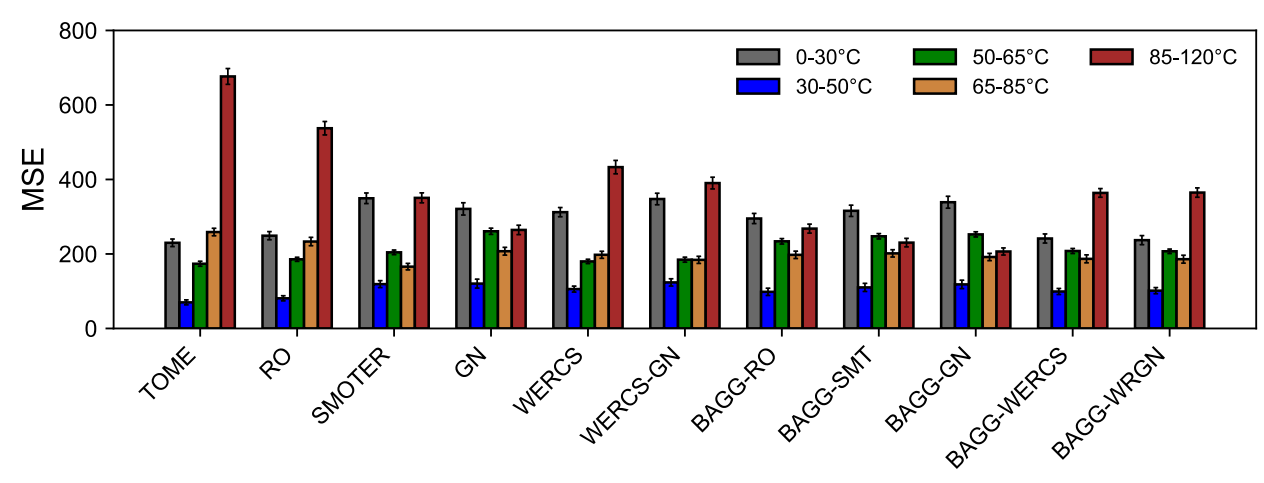

$\mathbf{E}$

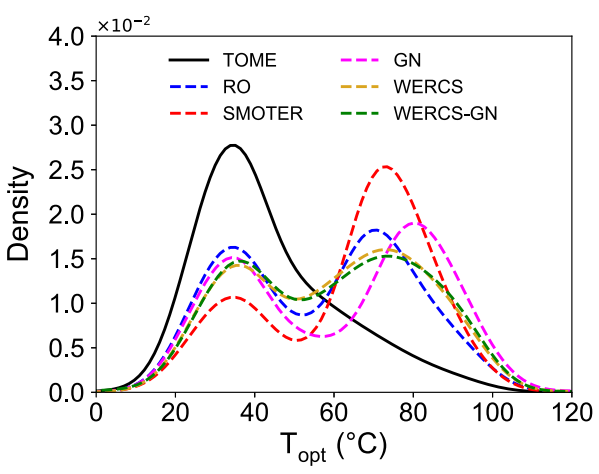

$\mathbf{F}$

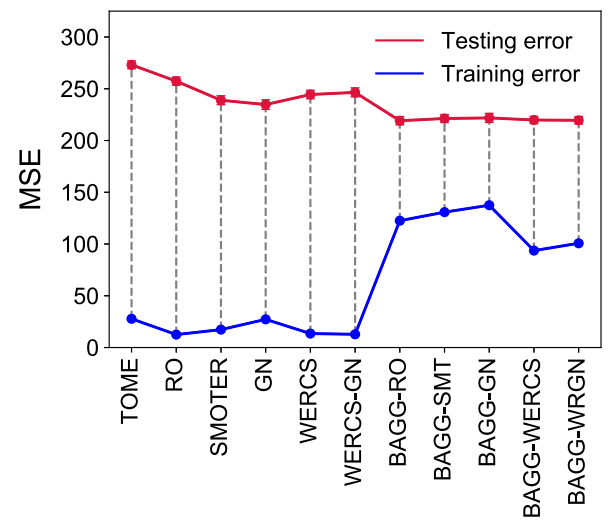

Figure 2. Performance of the resampling strategies. The resampling strategies were applied to the training dataset, regressors were fitted on the resampled data, and the performance was evaluated on a uniformly distributed test set with 50 iterations of Monte Carlo cross validation. Error bars indicate $95 \%$ confidence interval of the mean over 50 iterations. (A) Highest $R^{2}$ value of the resampling strategies determined from a grid search of hyperparameter combinations. Combining bagging with the resampling strategies via the REBAGG algorithm outperforms the resampling strategies alone. See Figure S1 for the performance of all hyperparameter combinations. (B) MCC and (C) $F_{1}$ scores of the best hyperparameter combinations of the resampling strategies, i.e., combinations that yielded the highest $\mathrm{R}^{2}$ value. (D) Mean squared error on different ranges of the target values. Without resampling (TOME), the error is highest in the $85-120^{\circ} \mathrm{C}$ range, but all the resampling strategies significantly reduce this error. The lowest overall error is achieved by the BAGG-RO strategy. (E) Distribution (KDE) of the dataset after applying the resampling methods with optimal hyperparameters. (F) Mean squared error when regressors trained on resampled data are applied to the training set and the testing set. The integration of resampling strategies with bagging decreases the variance as shown by an increase in training error and decrease in testing error. 
Analysis of the MSE as a function of the true $T_{\text {opt }}$ values indicates that there is significant variation in the MSE across the range of target values (Figure 2D). Without resampling (TOME), the error inversely correlates with the frequency of the data, with lower error in regions of abundant data $\left(30-50^{\circ} \mathrm{C}\right)$ and higher error in regions of rare data $\left(0-30^{\circ} \mathrm{C}, 65-120^{\circ} \mathrm{C}\right)$. Moreover, error in the $65-85^{\circ} \mathrm{C}$ and $85-120^{\circ} \mathrm{C}$ ranges was 3.7 and 9.7 times higher, respectively, than the error in the $30-50^{\circ} \mathrm{C}$ range. Hence, without resampling, the regressor (TOME) overfits to abundant values and demonstrates inferior performance on high temperature values. In applications that rely on TOME for identifying high $\mathrm{T}_{\text {opt }}$ enzymes, the large error on high temperature values may lead to misleading results. By applying resampling strategies to the training set, we altered the distribution of the training dataset to prevent the learning algorithm from overfitting to abundant values and to improve performance on rare high temperature values (Figure 2E). As Figure 2D shows, all the resampling strategies led to a reduction of the error in the high temperature ranges $\left(65-120^{\circ} \mathrm{C}\right)$ and an increase of the error in the abundant data range $\left(30-50^{\circ} \mathrm{C}\right)$, which indicates a decrease in the overfitting of abundant values. Moreover, the error in the abundant data range is the lowest error for TOME as well as for all the resampling strategies. This suggests that there is an upper limit to the performance gain from resampling rare data, and more experimental data which sample unexplored regions of the rare data space may be necessary for further improvement in performance.

Furthermore, the combination of resampling methods with bagging, such that each base regressor was trained on independently resampled datasets, yielded significantly higher overall performance scores $\left(\mathrm{R}^{2}\right.$ and $\mathrm{MCC}$ ) than resampling methods alone (Figure 2A and $\mathrm{B}$ ). Other researchers have similarly observed that ensemble learning methods, such as bagging and boosting, considerably enhance the effect of resampling techniques. ${ }^{34,39,58-60}$ In this work, the resampling methods without bagging (i.e., RO, SMOTER, GN, WERCS, and WERCS-GN) simply increased the proportion of rare values (Figure 2E), which decreased the overfitting of the regressors to abundant data, and, consequently, led to a reduction of both training error and testing error (Figure 2F). However, the difference between the testing and training error was substantial, indicating that the regressors were overfitting to the resampled training data (high variance). On the other hand, when the resampling methods were repeatedly applied to multiple decision trees in an ensemble (i.e., the REBAGG strategies) such that each base tree was trained on differently sampled datasets, a much lower testing error and a higher training error was observed. This outcome indicates that the integration of bagging with the resampling methods (i.e., BAGG-RO, BAGG-SMT, BAGG-WERCS, and BAGG-WRGN) reduces the variance of individual regressors and prevents overfitting to the resampled training data, leading to improved generalization. ${ }^{61}$ Moreover, all REBAGG strategies yielded similar overall performance $\left(R^{2}\right.$ and $\left.M C C\right)$, which suggests that the specific resampling method applied in the REBAGG strategy had little effect on the overall performance. The BAGG-RO strategy led to the highest $R^{2}$ value of 0.632 and the lowest MSE of 218.6.

\subsection{Effect of base learners on ensemble performance}

We examined the influence of different base learners in the BAGG-RO ensemble to assess whether further performance enhancement could be attained. Using the optimal resampling hyperparameters determined with decision trees (Table 3), we applied four additional base regressors in the BAGG-RO ensemble: support vector regressor (SVR), $k$-neighbor regressor (KNR), elastic net (ENET) regressor, and Bayesian ridge regressor (BAYR). For each of these regressors, we used a grid search to determine optimal hyperparameters that yielded the best $\mathrm{R}^{2}$ value (Table 4), and the performance was measured as an average over $50 \mathrm{MCCV}$ iterations. The results indicate that, although each alternative regressor outperformed TOME, the decision tree base regressor yielded the highest $\mathrm{R}^{2}$ value and lowest overall MSE. Interestingly, the decision tree regressor showed the lowest $F_{1}$ score (Figure 3). These results suggest that, while other regressors possibly perform better on high temperature values, tree-based 
A

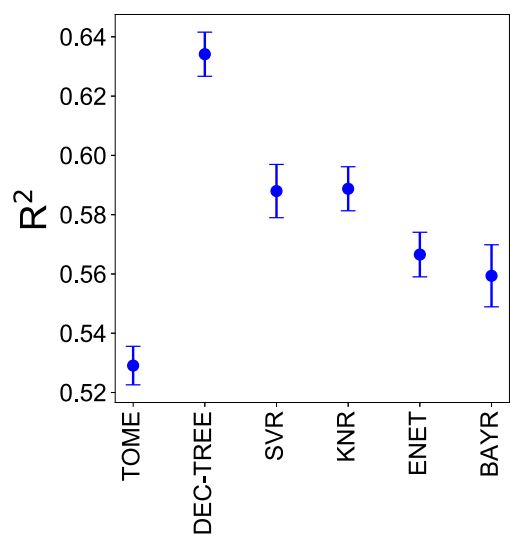

B

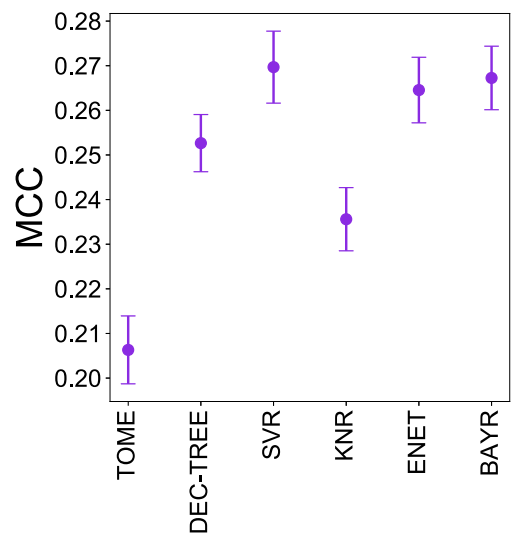

C

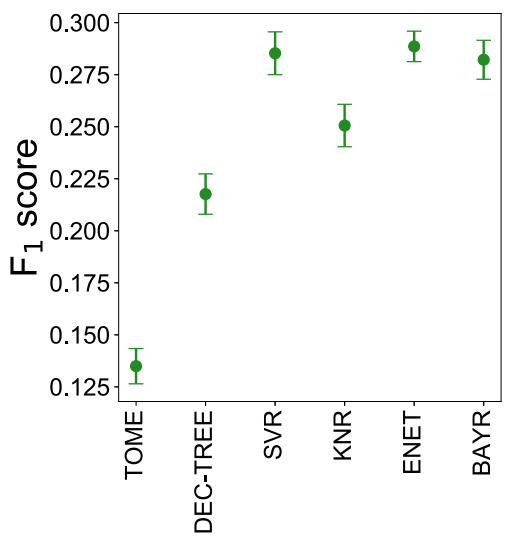

Figure 3. Performance of BAGG-RO ensemble with different base learners. The optimal hyperparameters for the base learners were determined by a grid search. (A) Highest $R^{2}$ value achieved for different base learners in the BAGG-RO ensemble. (B) Matthew's correlation coefficient and (C) $F_{1}$ scores of BAGG-RO strategy with different base learners using the optimal hyperparameters, i.e., hyperparameters that yielded the highest $R^{2}$ value.

Table 4: Hyperparameters for base learners in BAGG-RO ensemble

\begin{tabular}{|l|l|l|}
\hline Base learner & Hyperparameter range & $\begin{array}{l}\text { Optimal } \\
\text { hyperparameters }\end{array}$ \\
\hline $\begin{array}{l}\text { Support vector } \\
\text { regressor (SVR) }\end{array}$ & $\begin{array}{l}\mathrm{C}=\left[10^{-3}, 10^{-2}, 10^{-1}, 10^{0}, 10^{1}, 10^{2}\right], \\
\text { gamma }=\left[10^{-3}, 10^{-2}, 10^{-1}, 10^{0}, 10^{1}, 10^{2}\right]\end{array}$ & $\begin{array}{l}\mathrm{C}=10^{2}, \\
\text { gamma }=10^{-2}\end{array}$ \\
\hline $\begin{array}{l}\mathrm{k} \text {-neighbor } \\
\text { regressor (KNR) }\end{array}$ & $\mathrm{k}=[3,5,7,10,15,20,30]$ & $\mathrm{k}=3$ \\
\hline $\begin{array}{l}\text { Elastic net } \\
\text { regressor (ENET) }\end{array}$ & alpha $=\left[10^{-3}, 10^{-2}, 10^{-1}, 10^{0}, 10^{1}, 10^{2}\right]$ & alpha $=10^{-2}$ \\
\hline $\begin{array}{l}\text { Bayesian ridge } \\
\text { regressor (BAYR) }\end{array}$ & None (default) & None (default) \\
\hline
\end{tabular}

regressors exhibit the best overall performance in predicting $T_{\text {opt }}$ values from amino acid composition and OGT. ${ }^{23,26}$

\subsection{Final model, data and code availability}

We identified the BAGG-RO strategy with decision tree base learners as the optimal resampling strategy for predicting enzyme optimum temperatures across the entire range of experimental $T_{\text {opt }}$ values because it led to the highest $\mathrm{R}^{2}$ value and lowest overall MSE. A final model was prepared by applying the BAGG-RO resampling strategy with optimal hyperparameters (Table 3) to the entire dataset of 2,917 proteins. The final model is available to researchers as a Python package, TOMER (Temperature Optima for Enzymes with Resampling), on the Python package index, http://pypi.org/project/tomer/ with the source code publicly available at http://github.com/jafetgado/tomer/. Compared to TOME, TOMER provides a $20 \%$ improvement in the overall predictive performance $\left(R^{2}\right)$, and a $25 \%$ and $60 \%$ decrease in MSE on $\mathrm{T}_{\text {opt }}$ values in the $65-85^{\circ} \mathrm{C}$ and $85-120^{\circ} \mathrm{C}$ ranges, respectively. All data and code used and produced in this study are available at https://github.com/jafetgado/tomerdesign/. We have also prepared a Python package, resreg (resampling for regression), for applying the resampling strategies discussed in this work to other regression problems. It is available on the Python repository, http://pypi.org/project/resreg, with the source code at http://github.com/jafetgado/resreg. 


\section{CONCLUSIONS}

In this study, we applied resampling strategies to improve the performance of predicting enzyme optimum temperatures with machine learning. The resampling strategies were implemented to modify the imbalanced distribution of the training set and improve performance on regions with sparse data. Compared with TOME, which at the time of this study is the only available machine-learning tool for predicting enzyme optimum temperatures, our method (TOMER) yields a significant improvement in predictive accuracy, particularly in the thermophilic regimes. We expect that TOMER will find useful application in high-throughput

prospecting of enzymes that are both stable and active at high temperatures. TOMER requires the user to provide the amino acid sequence of the enzyme and the OGT of the source organism. If the OGT is unknown, it may be predicted using TOME. ${ }^{23}$ For future considerations, the incorporation of higherlevel features or the addition of more experimental data may prove useful strategies for further improving the performance of TOMER. Ultimately, this study highlights the critical need to consider data imbalance in regression problems, especially when the rare, extreme data range is of greater scientific interest than the abundant data region. We anticipate that our Python tool for readily implementing resampling strategies in regression problems (resreg) will be a valuable resource for other researchers in dealing with the challenges of data imbalance.

\section{ASSOCIATED CONTENT}

\section{Supporting Information}

Figure S1, performance of resampling strategies for all hyperparameter combinations

Algorithms (pseudocode) for the RO, SMOTER, GN, WERCS, WERCS-GN, and REBAGG resampling strategies.

\section{AUTHOR INFORMATION}

\section{Corresponding Authors}

E-mail: christy.payne@uky.edu

E-mail: gregg.beckham@nrel.gov

\section{ORCID}

Japheth E. Gado: 0000-0002-0024-2531

Gregg T. Beckham: 0000-0002-3480-212X

Christina M. Payne: 0000-0001-5264-0964

\section{Notes}

The authors declare no competing financial interest.

\section{ACKNOWLEDGMENTS}

This work was partially supported by the National Science Foundation (CBET-1552355 to CMP in support of JEG). Support for GTB was provided by the U.S. Department of Energy, Office of Energy Efficiency and Renewable Energy, Advanced Manufacturing Office (AMO) and Bioenergy Technologies Office (BETO). This work was performed as part of the BOTTLE ${ }^{\text {TM }}$ Consortium and was supported by 
$\mathrm{AMO}$ and BETO under contract no. DE-AC36-08GO28308 with the National Renewable Energy Laboratory, operated by Alliance for Sustainable Energy, LLC. This material is also based upon work supported by (while CMP is serving at) the NSF. Any opinion, findings, and conclusions or recommendations expressed in this material are those of the authors and do not necessarily reflect the views of the NSF. The authors also thank Dr. Peter St. John (NREL) and Dr. Brent Harrison (University of Kentucky) for a critical reading of the manuscript.

\section{REFERENCES}

1. Ku, T.; Lu, P.; Chan, C.; Wang, T.; Lai, S.; Lyu, P.; Hsiao, N., Predicting melting temperature directly from protein sequences. Comput. Biol. Chem. 2009, 33, 445-450.

2. Gorania, M.; Seker, H.; Haris, P. I., Predicting a protein's melting temperature from its amino acid sequence. Conf. Proc. IEEE. Eng. Med. Biol. Soc. 2010, 1820-3.

3. Pucci, F.; Dhanani, M.; Dehouck, Y.; Rooman, M., Protein thermostability prediction within homologous families using temperature-dependent statistical potentials. PloS One 2014, 9.

4. Capriotti, E.; Fariselli, P.; Casadio, R., A neural-network-based method for predicting protein stability changes upon single point mutations. Bioinformatics 2004, 20, i63-i68.

5. Cheng, J.; Randall, A.; Baldi, P., Prediction of protein stability changes for single-site mutations using support vector machines. Proteins 2006, 62, 1125-1132.

6. Montanucci, L.; Fariselli, P.; Martelli, P. L.; Casadio, R., Predicting protein thermostability changes from sequence upon multiple mutations. Bioinformatics 2008, 24, i190-5.

7. Dehouck, Y.; Grosfils, A.; Folch, B.; Gilis, D.; Bogaerts, P.; Rooman, M., Fast and accurate predictions of protein stability changes upon mutations using statistical potentials and neural networks: PoPMuSiC-2.0. Bioinformatics 2009, 25, 2537-43.

8. Li, Y.; Fang, J., PROTS-RF: a robust model for predicting mutation-induced protein stability changes. PLoS One 2012, 7, e47247.

9. Berliner, N.; Teyra, J.; Colak, R.; Garcia Lopez, S.; Kim, P. M., Combining structural modeling with ensemble machine learning to accurately predict protein fold stability and binding affinity effects upon mutation. PLoS One 2014, 9, e107353.

10. Pucci, F.; Bourgeas, R.; Rooman, M., Predicting protein thermal stability changes upon point mutations using statistical potentials: Introducing HoTMuSiC. Sci. Rep. 2016, 6, 23257.

11. Cao, H.; Wang, J.; He, L.; Qi, Y.; Zhang, J. Z., DeepDDG: Predicting the Stability Change of Protein Point Mutations Using Neural Networks. J. Chem. Inf. Model. 2019, 59, 1508-1514.

12. Alvarez-Machancoses, O.; De Andres-Galiana, E. J.; Fernandez-Martinez, J. L.; Kloczkowski, A., Robust Prediction of Single and Multiple Point Protein Mutations Stability Changes. Biomolecules 2020, 10.

13. Zhang, G.; Fang, B., Support vector machine for discrimination of thermophilic and mesophilic proteins based on amino acid composition. Protein Pept. Lett. 2006, 13, 965-70.

14. Gromiha, M. M.; Suresh, M. X., Discrimination of mesophilic and thermophilic proteins using machine learning algorithms. Proteins 2008, 70, 1274-9.

15. Wu, L.-C.; Lee, J.-X.; Huang, H.-D.; Liu, B.-J.; Horng, J.-T., An expert system to predict protein thermostability using decision tree. Expert Syst. Appl. 2009, 36, 9007-9014.

16. Li, Y.; Middaugh, C. R.; Fang, J., A novel scoring function for discriminating hyperthermophilic and mesophilic proteins with application to predicting relative thermostability of protein mutants. $B M C$ Bioinform. 2010, 11, 62.

17. Lin, H.; Chen, W., Prediction of thermophilic proteins using feature selection technique. J. Microbiol. Methods 2011, 84, 67-70. 
18. Ebrahimi, M.; Lakizadeh, A.; Agha-Golzadeh, P.; Ebrahimie, E.; Ebrahimi, M., Prediction of thermostability from amino acid attributes by combination of clustering with attribute weighting: a new vista in engineering enzymes. PLoS One 2011, 6, e23146.

19. Jensen, D. B.; Vesth, T. C.; Hallin, P. F.; Pedersen, A. G.; Ussery, D. W., Bayesian prediction of bacterial growth temperature range based on genome sequences. BMC Genomics 2012, 13 Suppl 7, S3. 20. Zuo, Y. C.; Chen, W.; Fan, G. L.; Li, Q. Z., A similarity distance of diversity measure for discriminating mesophilic and thermophilic proteins. Amino Acids 2013, 44, 573-80.

21. Fan, G. L.; Liu, Y. L.; Wang, H., Identification of thermophilic proteins by incorporating evolutionary and acid dissociation information into Chou's general pseudo amino acid composition. J. Theor. Biol. 2016, 407, 138-142.

22. Dehouck, Y.; Folch, B.; Rooman, M., Revisiting the correlation between proteins' thermoresistance and organisms' thermophilicity. Protein Eng. Des. Sel. 2008, 21, 275-8.

23. Li, G.; Rabe, K. S.; Nielsen, J.; Engqvist, M. K. M., Machine Learning Applied to Predicting Microorganism Growth Temperatures and Enzyme Catalytic Optima. ACS Synth. Biol. 2019, 8, 14111420.

24. Nakashima, H.; Fukuchi, S.; Nishikawa, K., Compositional changes in RNA, DNA and proteins for bacterial adaptation to higher and lower temperatures. J. Biochem. 2003, 133, 507-13.

25. Zeldovich, K. B.; Berezovsky, I. N.; Shakhnovich, E. I., Protein and DNA sequence determinants of thermophilic adaptation. PLoS Comput. Biol. 2007, 3, e5.

26. Li, G.; Zrimec, J.; Ji, B.; Geng, J.; Larsbrink, J.; Zelezniak, A.; Nielsen, J.; Engqvist, M. K. M., Performance of regression models as a function of experiment noise. arXiv:.1912.081412019.

27. Chen, Z.; Zhao, P.; Li, F.; Leier, A.; Marquez-Lago, T. T.; Wang, Y.; Webb, G. I.; Smith, A. I.; Daly, R. J.; Chou, K. C.; Song, J., iFeature: a Python package and web server for features extraction and selection from protein and peptide sequences. Bioinformatics 2018, 34, 2499-2502.

28. Alley, E. C.; Khimulya, G.; Biswas, S.; AlQuraishi, M.; Church, G. M., Unified rational protein engineering with sequence-based deep representation learning. Nat. Methods 2019, 16, 1315-1322.

29. He, H.; Garcia, E. A., Learning from imbalanced data. IEEE T. Knowl. Data. En. 2009, 21, 12631284.

30. Krawczyk, B., Learning from imbalanced data: open challenges and future directions. Prog. Artif. Intell. 2016, 5, 221-232.

31. López, V.; Fernández, A.; García, S.; Palade, V.; Herrera, F., An insight into classification with imbalanced data: Empirical results and current trends on using data intrinsic characteristics. Inf. Sci 2013, 250, 113-141.

32. Branco, P.; Torgo, L.; Ribeiro, R. P., A survey of predictive modeling on imbalanced domains. ACM Comput. Surv. 2016, 49, 1-50.

33. Elrahman, S. M. A.; Abraham, A., A review of class imbalance problem. J. Netw. Innov. Comput. 2013, 1, 332-340.

34. Galar, M.; Fernandez, A.; Barrenechea, E.; Bustince, H.; Herrera, F., A review on ensembles for the class imbalance problem: bagging-, boosting-, and hybrid-based approaches. IEEE T. Syst. Man Cy. C 2012, 42, 463-484.

35. Laurikkala, J. Improving identification of difficult small classes by balancing class distribution. In Conference on Artificial Intelligence in Medicine in Europe, 2001; Springer: 2001; pp 63-66.

36. Chawla, N. V.; Bowyer, K. W.; Hall, L. O.; Kegelmeyer, W. P., SMOTE: synthetic minority oversampling technique. J. Artif. Intell. Res. 2002, 16, 321-357.

37. Stefanowski, J.; Wilk, S. Selective pre-processing of imbalanced data for improving classification performance. In International Conference on Data Warehousing and Knowledge Discovery, 2008; Springer: 2008; pp 283-292. 
38. Bunkhumpornpat, C.; Sinapiromsaran, K.; Lursinsap, C. Mute: Majority under-sampling technique. In 8th International Conference on Information, Communications \& Signal Processing, 2011; IEEE: 2011; pp 1-4.

39. Seiffert, C.; Khoshgoftaar, T. M.; Van Hulse, J.; Napolitano, A., RUSBoost: A hybrid approach to alleviating class imbalance. IEEE Trans. Syst., Man, Cybern. Syst. 2010, 40, 185-197.

40. Zhang, Y.; Zhang, D.; Mi, G.; Ma, D.; Li, G.; Guo, Y.; Li, M.; Zhu, M., Using ensemble methods to deal with imbalanced data in predicting protein-protein interactions. Comput. Biol. Chem. 2012, 36, 3641.

41. Torgo, L.; Branco, P.; Ribeiro, R. P.; Pfahringer, B., Resampling strategies for regression. Expert Syst. 2015, 32, 465-476.

42. Branco, P.; Torgo, L.; Ribeiro, R. P. SMOGN: a Pre-processing Approach for Imbalanced Regression. In First International Workshop on Learning with Imbalanced Domains: Theory and Applications, 2017; 2017; pp 36-50.

43. Branco, P.; Torgo, L.; Ribeiro, R. P. MetaUtil: Meta learning for utility maximization in regression. In International Conference on Discovery Science, 2018; Springer: 2018; pp 129-143.

44. Branco, P.; Torgo, L.; Ribeiro, R. P. Rebagg: Resampled bagging for imbalanced regression. In Second International Workshop on Learning with Imbalanced Domains: Theory and Applications, 2018; 2018; pp 67-81.

45. Branco, P.; Torgo, L.; Ribeiro, R. P., Pre-processing approaches for imbalanced distributions in regression. Neurocomputing 2019, 343, 76-99.

46. Stepniewska-Dziubinska, M. M.; Zielenkiewicz, P.; Siedlecki, P., Development and evaluation of a deep learning model for protein-ligand binding affinity prediction. Bioinformatics 2018, 34, 3666-3674.

47. Sharma, A. K.; Srivastava, G. N.; Roy, A.; Sharma, V. K., ToxiM: A Toxicity Prediction Tool for Small Molecules Developed Using Machine Learning and Chemoinformatics Approaches. Front. Pharmacol. 2017, 8, 880 .

48. Torgo, L.; Ribeiro, R. Precision and recall for regression. In International Conference on Discovery Science, 2009; Springer: 2009; pp 332-346.

49. Jeske, L.; Placzek, S.; Schomburg, I.; Chang, A.; Schomburg, D., BRENDA in 2019: a European ELIXIR core data resource. Nucleic Acids Res. 2019, 47, D542-D549.

50. Pedregosa, F.; Varoquaux, G.; Gramfort, A.; Michel, V.; Thirion, B.; Grisel, O.; Blondel, M.; Prettenhofer, P.; Weiss, R.; Dubourg, V., Scikit-learn: Machine learning in Python. J. Mach. Learn. Res. 2011, 12, 2825-2830.

51. Molinaro, A. M.; Simon, R.; Pfeiffer, R. M., Prediction error estimation: a comparison of resampling methods. Bioinformatics 2005, 21, 3301-7.

52. Matthews, B. W., Comparison of the predicted and observed secondary structure of T4 phage lysozyme. Biochim. Biophys. Acta 1975, 405, 442-51.

53. Gorodkin, J., Comparing two K-category assignments by a K-category correlation coefficient. Comput. Biol. Chem. 2004, 28, 367-74.

54. Torgo, L.; Ribeiro, R. Utility-based regression. In European Conference on Principles of Data Mining and Knowledge Discovery, 2007; Springer: 2007; pp 597-604.

55. Branco, P.; Ribeiro, R. P.; Torgo, L., UBL: an R package for utility-based learning. arXiv preprint arXiv:1604.08079 2016.

56. Breiman, L., Bagging predictors. Mach. Learn. 1996, 24, 123-140.

57. Menardi, G.; Torelli, N., Training and assessing classification rules with imbalanced data. Data Min. Knowl. Disc. 2014, 28, 92-122. 
58. Chawla, N. V.; Lazarevic, A.; Hall, L. O.; Bowyer, K. W. SMOTEBoost: Improving prediction of the minority class in boosting. In European conference on principles of data mining and knowledge discovery, 2003; Springer: 2003; pp 107-119.

59. Wang, S.; Yao, X. Diversity analysis on imbalanced data sets by using ensemble models. In IEEE Symposium on Computational Intelligence and Data Mining, 2009; IEEE: 2009; pp 324-331.

60. Błaszczyński, J.; Deckert, M.; Stefanowski, J.; Wilk, S. Integrating selective pre-processing of imbalanced data with ivotes ensemble. In International conference on rough sets and current trends in computing, 2010; Springer: 2010; pp 148-157.

61. Buja, A.; Stuetzle, W., The effect of bagging on variance, bias, and mean squared error. Preprint. AT\&T Labs-Research 2000.

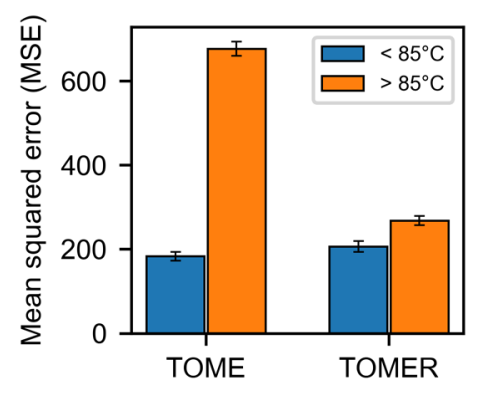

Figure: For Table of Contents Only 